\title{
Einkommensverhältnisse der freien Ärzteschaft der Schweiz in den Jahren 2003 (neu) und 2002 (Re-Evaluation)
}

Niklaus Hasler

Gutachten erstellt im Auftrag der Verbindung der Schweizer Ärztinnen und Ärzte FMH. Diese Artikel kann im Internet über die Homepage der FMH www.fmh.ch $\rightarrow$ Deutsch $\rightarrow$ Über uns $\rightarrow$ Ärzteeinkommen abgerufen werden. Die Numerierung der Seiten, Tabellen und Abbildungen bezieht sich auf den vollständigen Bericht. Das vollständige Gutachten mit 61 Seiten, 28 Tabellen und 30 Abbildungen ist auf Anfrage unter folgender Adresse erhältlich: kommunikation@ fmh.ch.

Korrespondenz:

Dr. med. Niklaus Hasler Huzlenstrasse 3

CH-8604 Volketswil

nik@hin.ch

\begin{abstract}
A. Einleitung
Mit Datum vom 11. Juni 2006 hat die Verbindung der Schweizer Ärztinnen und Ärzte den Auftrag erteilt, die Einkommensverhältnisse der freien Ärzteschaft der Schweiz für das Einkommensjahr 2003 erneut durch eine Vollerhebung $\mathrm{zu}$ analysieren.
\end{abstract}

\section{A. 1 Bemerkung zu TARMED}

Der grundsätzlich neue, gesamtschweizerisch gültige Arzttarif wurde für die Behandlungen nach Unfallversicherungsgesetz, nach Invalidenversicherung und nach Militärversicherungsgesetz auf den 1. Mai 2003 eingeführt.

Nach langjährigen Statistiken machen die Arztrechnungen an die Versicherer nach Unfallversicherungsgesetz, an die Invalidenversicherung und an die Militärversicherung rund 8 bis $10 \%$ des gesamten Honorarvolumens der schweizerischen Ärzteschaft in freier Praxis aus. Da der TARMED nur während 8 Monaten des Jahres 2003 Pflichtgrundlage zur Abrechnung war, sind rund 5,3 bis $6,7 \%$ des gesamten, in diesem Gutachten für das Jahr 2003 berücksichtigten Honorarvolumina aufgrund von TARMED abgerechnet.

Das im Oktober 2005 publizierte Einkommensgutachten der Ärzteeinkommen des Jahres 2002 war somit das letzte Gutachten, das vollständig ohne Anwendung des TARMED-Tarifs erhoben wird.

Das nächste Gutachten der Einkommen des Jahres 2004 (erscheint in der zweiten Hälfte 2007) umfasst Bruttoeinkünfte, die alle nach TARMED-Tarif abgerechnet worden sind.

Die Erhebung schliesst direkt an die früheren 21 Berichte an, die zwischen August 1977 und Oktober 2005 erstellt wurden.

Hier folgt eine Zusammenfassung der wichtigsten Resultate, Tabellen und Abbildungen. In dieser werden bewusst nur die Zahlen für die Ärzte unter 66 Jahren angegeben. In der Vollversion sind jeweils auch die Zahlen für die Ärzte über 65 Jahre und die Zahlen für alle Ärzte (unabhängig vom Alter) angegeben.

Zudem werden in der Vollversion des Gutachtens noch weitere Kriterien genau untersucht, wie z.B. die Abhängigkeit des Einkommens von der Personallohnsumme, vom Alter des Arztes oder von der Zeit in Jahren seit der Praxiseröffnung.

Im nächsten Kapitel wird die wichtigste Änderung der Erhebung beschrieben, die in den Zeitraum 1997/98 bis 2001 fällt, nämlich der Übergang von der Vergangenheitsbesteuerung auf die Gegenwartsbesteuerung.

\section{A.2 Übergang auf die Gegenwarts- besteuerung}

Im Rahmen der eidgenössischen Steuerharmonisierung wurde auch die Veranlagung der AHVBeiträge der Selbständigerwerbenden von der Vergangenheitsbesteuerung auf die Gegenwartsbesteuerung umgestellt.

Das Durchschnittseinkommen der Jahre 1997 und 1998 (30. Wehrsteuerperiode) ist die Grundlage für die Verfügung der in den Jahren 1999 und $2000 \mathrm{zu}$ bezahlenden persönlichen AHVBeiträge.

Die Berufseinkommen der Jahre 1999 und 2000 fallen in die Bemessungslücke und können deshalb nicht erhoben und analysiert werden.

Ab dem Jahr 2001 gilt die Gegenwartsbesteuerung, d.h. das Einkommensjahr 2001 entspricht auch dem AHV-Beitragsjahr 2001.

Bis zu den Einkommen der 30. Wehrsteuerperiode (Einkommensjahre 1997/98) konnten die Ärzteeinkommen nur als zweijähriger Mittelwert im Zwei-Jahres-Rhythmus erhoben werden.

Die Gegenwartsbesteuerung erlaubt nun, die Einkommensverhältnisse der freien Ärzteschaft der Schweiz in jährlichem Rhythmus zu analysieren, was eine raschere und engmaschigere Beurteilung zulässt. 


\section{Resultate}

\section{D.1 Allgemeines}

Zur Überprüfung der im Oktober 2005 publizierten Einkommenszahlen 2002 wurden diese Werte in der vorliegenden Erhebung erneut erhoben und analysiert. Die revidierten, jetzt publizierten Werte liegen durchwegs leicht tiefer, aber doch sehr nahe an den Werten der Erhebung vom Oktober 2005.

In der Vollversion des Gutachtens sind einige Beispiele angegeben.

Genauso wie die absoluten Werte für die Einkommen 2002 müssen die Veränderungen der Einkommen zwischen 2001 und 2002 - wie in der Vollversion des Gutachtens angegeben - korrigiert werden.

\section{D.2 Einkommensentwicklung 2002 bis 2003}

Der Anstieg der Teuerung zwischen 2002 und 2003 betrug $+0,67 \%$. Im gleichen Zeitraum ist das mittlere Ärzteeinkommen (AHV-pflichtiges Berufseinkommen) für folgende Teilgesamtheiten der freien Ärzteschaft der Schweiz wie folgt abgesunken bzw. angestiegen:

- alle Ärzte: von 207140 auf 200549 um $-3,18 \%$;

- Ärzte unter 66 Jahren: von 214362 auf 207430 um $-3,23 \%$;

- Ärzte über 65 Jahre: von 90821 auf 91917 um $+1,21 \%$.

Das zentrale Ärzteeinkommen (das Einkommen der einen Hälfte der angegebenen Ärzte liegt unter, das Einkommen der anderen Hälfte der angegebenen Ärzte liegt über dem zentralen Ärzteeinkommen) ist im gleichen Zeitraum für die gleichen Teilgesamtheiten wie folgt abgesunken bzw. angestiegen:

- alle Ärzte: von 166900 auf 161900 um $-3,00 \%$.

- Ärzte unter 66 Jahren: von 172000 auf 168000 um $-2,33 \%$;

- Ärzte über 65 Jahre: von 47100 auf 49200 um $+4,46 \%$.

Mit Ausnahme der Ärzte über 65 Jahre ist das mittlere Ärzteeinkommen zwischen 2002 und 2003 deutlich gesunken, währenddem die Teuerung angestiegen ist. Es ist ein reales Absinken von $-3,82$ bis $-3,87 \%$ festzustellen. Beim zentralen Ärzteeinkommen ist ein reales Absinken von $-3,65$ bis $-2,98 \%$ zu verzeichnen.

Bei den Ärzten über 65 Jahre ist beim mittleren Ärzteeinkommen ein realer Anstieg von $+0,54 \%$ und beim zentralen Ärzteeinkommen ein realer Zuwachs von $+3,76 \%$ festzustellen.
Für die Beurteilung der Tendenz könnte der Einbezug der über 65jährigen Ärzte mit Blick auf den zahlen- und einkommensmässig geringen Anteil vernachlässigt werden.

\section{D.3 Einkommensentwicklung 1971/72} bis 2003

Mit den revidierten Einkommenszahlen 2002 und den neuen Einkommenszahlen 2003 kann nun die Entwicklung des mittleren und des zentralen AHV-pflichtigen Einkommens der freien Ärzteschaft der Schweiz über gut drei Jahrzehnte verfolgt werden.

In den Abbildungen 13-16 (Anhang Seiten 53/54) sind die Indexreihen für das mittlere und das zentrale AHV-pflichtige Ärzteeinkommen (nur Ärzte unter 66 Jahren) sowohl nominal wie auch real (d. h. unter Berücksichtigung der Teuerung) dargestellt.

Der Tabelle 23 kann entnommen werden, dass im Zeitraum 1971/72 bis 2003 das mittlere AHV-pflichtige Ärzteeinkommen nominell um $60,4 \%$ und das zentrale AHV-pflichtige Ärzteeinkommen nominell nur um 46,2\% zugenommen hat. Wenn man aber die in der gleichen Zeit aufgelaufene Teuerung des LIKP von 155,4\% berücksichtigt, entspricht dieser Anstieg einem realen Verlust von 37,2 beim mittleren und von 42,8\% beim zentralen Ärzteeinkommen.

Von den vielen Tabellen und Abbildungen im Originalgutachten (via E-Mail zu bestellen bei kommunikation@fmh.ch) werden hier als Beispiele die Abbildungen 13 und 14, Entwicklung des Mittelwertes des AHV-pflichtigen Berufseinkommens der freien Ärzteschaft der Schweiz unter 66 Jahren nominal und real (unter Berücksichtigung der Teuerung), und die Tabellen 5 und 9 (Durchschnitt des AHV-pflichtigen Einkommens 2002 und 2003 der in freier Praxis tätigen unter 66 Jahre alten Ärzte nach Kantonen und nach Spezialitäten) gezeigt.

Bei der letzten Publikation des Einkommens 2002 im Januar 2006 in der Schweizerischen Ärztezeitung wurde mehrfach bemängelt, dass die entsprechenden Zentralwerte nicht publiziert wurden.

\section{D.4 Statistik}

Die Statistik ist die Wissenschaft, die Charakteristika einer Gesamtheit von Zahlen oder Werten durch möglichst wenige eindeutig definierte Werte darzustellen.

Die beiden gebräuchlichsten Werte sind der Mittelwert und der Zentralwert. Der Mittelwert oder Durchschnitt wird gebildet durch die Summe aller Einzelwerte dividiert durch die Anzahl Werte. 
Der Zentralwert oder Median ist der Wert, über dem die eine Hälfte aller Einzelwerte und unter dem die andere Hälfte der Einzelwerte liegen. Er kann nicht berechnet werden, sondern muss in der aufsteigenden Menge aller Einzelwerte ausgezählt werden.

Beispiel: Nehmen wir folgende 5 Einzelwerte: 100; 120; 150; 160 und 200. Der Mittelwert dieser Zahlenmenge beträgt 146 und der Zentralwert 150 .

Fügen wir nun zu dieser Zahlenmenge zwei weitere Zahlen, eine davon bewusst als Ausreisser, nämlich 140 und 990, dazu, dann erhalten wir die geordnete Zahlenmenge 100; 120; 140; 150; 160; 200 und 990.

Der Mittelwert dieser Zahlenmenge beträgt nun 266 und der Zentralwert 150.
Durch dieses Beispiel wird klar, dass der Mittelwert viel empfindlicher auf Ausreisser (gegen oben oder unten) reagiert als der Zentralwert.

Wie schon Prof. Dr. H. Riedwyl als bundesrätlicher Experte im Juni 1978 in seinem Gutachten festgestellt hat, lässt sich eine Gesamtheit von Zahlenwerten besser mit dem Zentralwert als mit dem Mittelwert charakterisieren.

Aus diesem Grunde werden neu die Abbildungen 15 und 16, Entwicklung 1971/72 bis 2003 des Zentralwertes des AHV-pflichtigen Berufseinkommens der freien Ärzteschaft der Schweiz unter 66 Jahren nominal und real (unter Berücksichtigung der Teuerung), und die Tabellen 7 und 13 (Zentralwert des AHV-pflichtigen Einkommens 2002 und 2003 der in freier Praxis tätigen unter 66 Jahre alten Ärzte nach Kantonen und nach Spezialitäten) gezeigt.

\section{Tabelle 23a}

Indexzahlen des AHV-pflichtigen Einkommens der freien Ärzteschaft der Schweiz (bis 1975/76 unter 63 Jahren, ab 1977/78 unter 66 Jahren). 1971/72 bis 2003: Erster Teil 1971/72 bis 1983/84. Alle Indexreihen sind auf der Basis 1971/72 = 100,0 berechnet.

Einkommensentwicklung: Indexzahlen (nur Ärzte unter 66 Jahren)

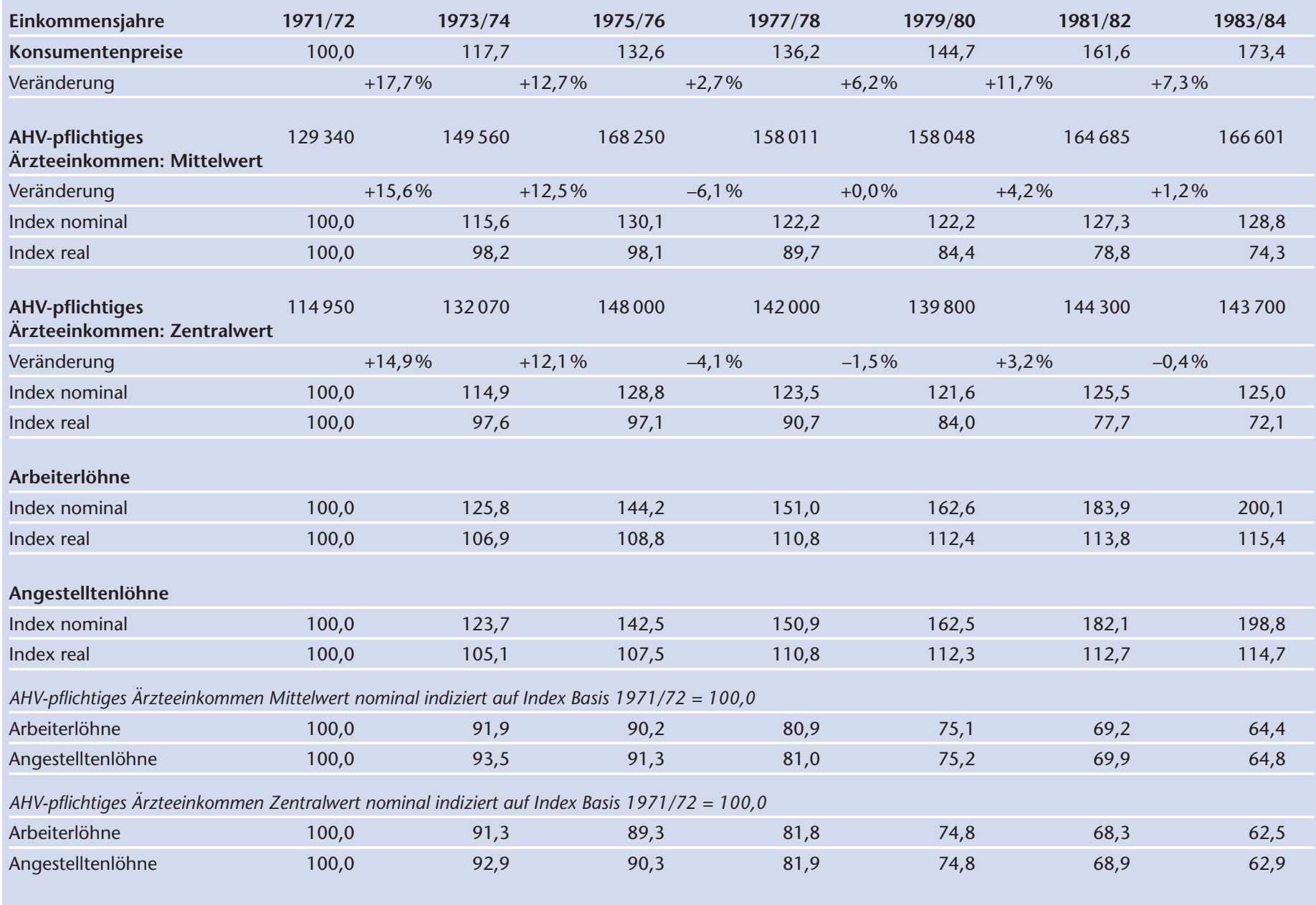




\section{D.5 Gesamtaufwand der Volkswirtschaft für die freie Ärzteschaft}

Es ist schwierig, den Gesamtaufwand der Volkswirtschaft für die freie Ärzteschaft in der Schweiz wirklich seriös zu recherchieren, da verschiedene Berechnungsfaktoren nur approximativ erhoben werden können (z. B. der Unkostensatz der Arztpraxis).

Für die Jahre 2002 und 2003 liegt der volkswirtschaftliche Gesamtaufwand für die freie Ärzteschaft der Schweiz um die 10,577 und 10,801 Mia Franken, was 2,46 bzw. 2,49\% des Bruttoinlandsproduktes BIP der Schweiz ausmacht.

\section{D.6 Tendenzen}

Die Ärzteeinkommen haben sich im Zeitraum 2002 bis 2003 bei einer geringen Steigerung des Lebenskostenindexes deutlich gesenkt. Das heisst, dass bei den Ärzteeinkommen in diesem Zeitraum ein reales Absinken festzustellen ist.

Generell kann festgehalten werden, dass in den letzten Jahren die Einkommen der operativ tätigen Ärzte etwas weniger rasch abgesunken sind als diejenigen der nichtoperativ tätigen Ärzte (= Grundversorger). Zudem ist festzustellen, dass in den letzten zwölf Jahren (siehe Kapitel D.20 auf Seiten 37/38) die Faktoren zwischen dem grössten und dem kleinsten Einkommen pro Kanton und pro Spezialität ebenfalls leicht abgesunken sind.

Es wird in den nächsten ein bis zwei Jahren interessant sein, die Auswirkungen des TARMEDTarifs zu beurteilen, der ja unter anderem zum Ziel hat, die operativ-technischen Leistungen zugunsten der ärztlich-intellektuellen Leistungen abzuwerten.

\section{Tabelle 23b}

Indexzahlen des AHV-pflichtigen Einkommens der freien Ärzteschaft der Schweiz (bis 1975/76 unter 63 Jahren, ab $1977 / 78$ unter 66 Jahren). $1971 / 72$ bis 2002: Zweiter Teil 1983/84 bis 1995/96. Alle Indexreihen sind auf der Basis 1971/72 = 100,0 berechnet.

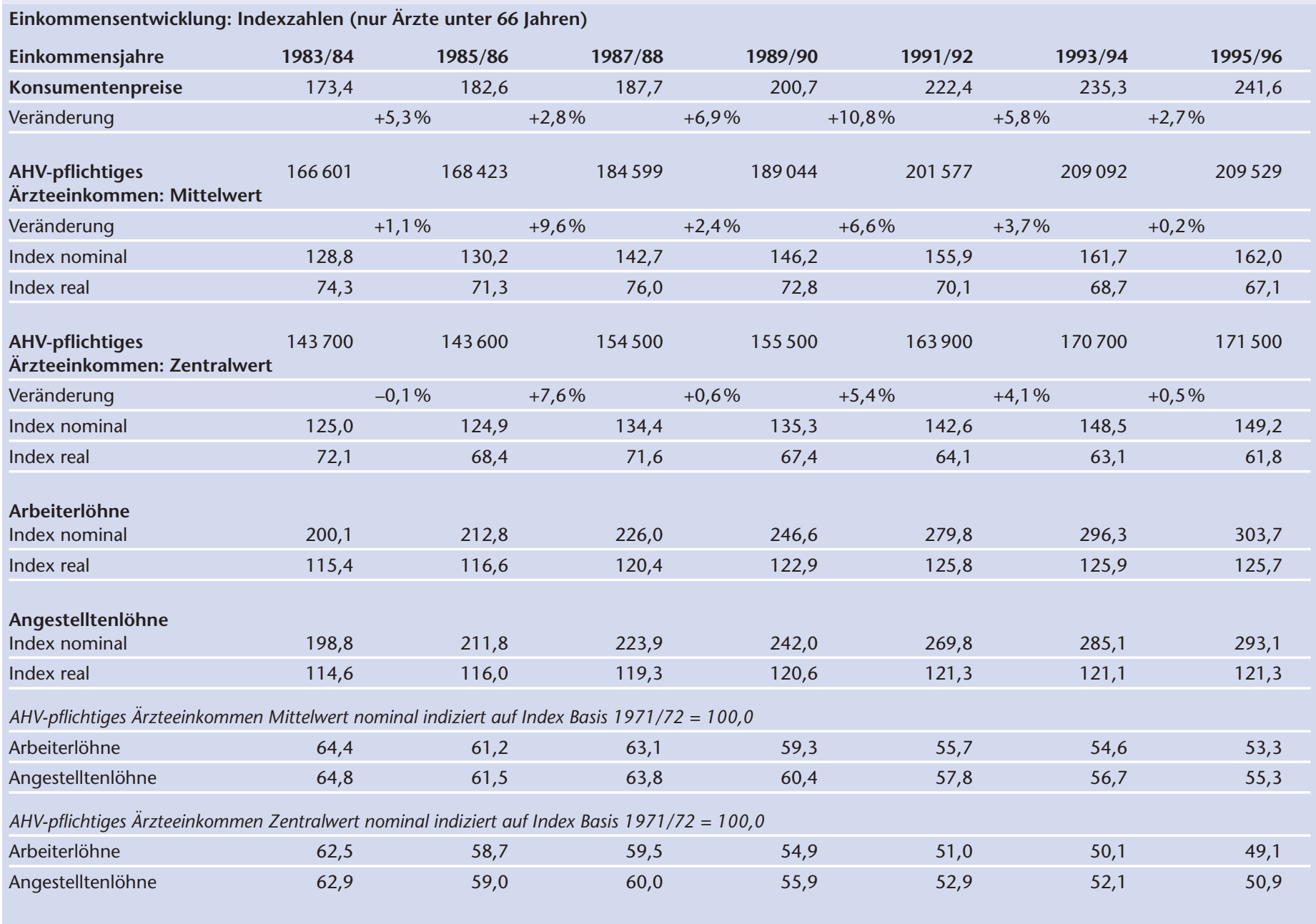


Die bisher erhobenen Zahlen für das Einkommensjahr 2003 scheinen zu bestätigen, dass der bisher immer festgestellte nominelle Daueranstieg der Ärzteeinkommen gebrochen ist.
Das Absinken der Ärzteeinkommen im Jahr 2003 darf noch nicht auf die Einführung des TARMEDTarifs zurückgeführt werden.

Tabelle 23c

Indexzahlen des AHV-pflichtigen Einkommens der freien Ärzteschaft der Schweiz (bis 1975/76 unter 63 Jahren, ab 1977/78 unter 66 Jahren). 1971/72 bis 2003: Dritter Teil 1995/96 bis 2003. Alle Indexreihen sind auf der Basis $1971 / 72=100,0$ berechnet.

Einkommensentwicklung: Indexzahlen (nur Ärzte unter 66 Jahren)

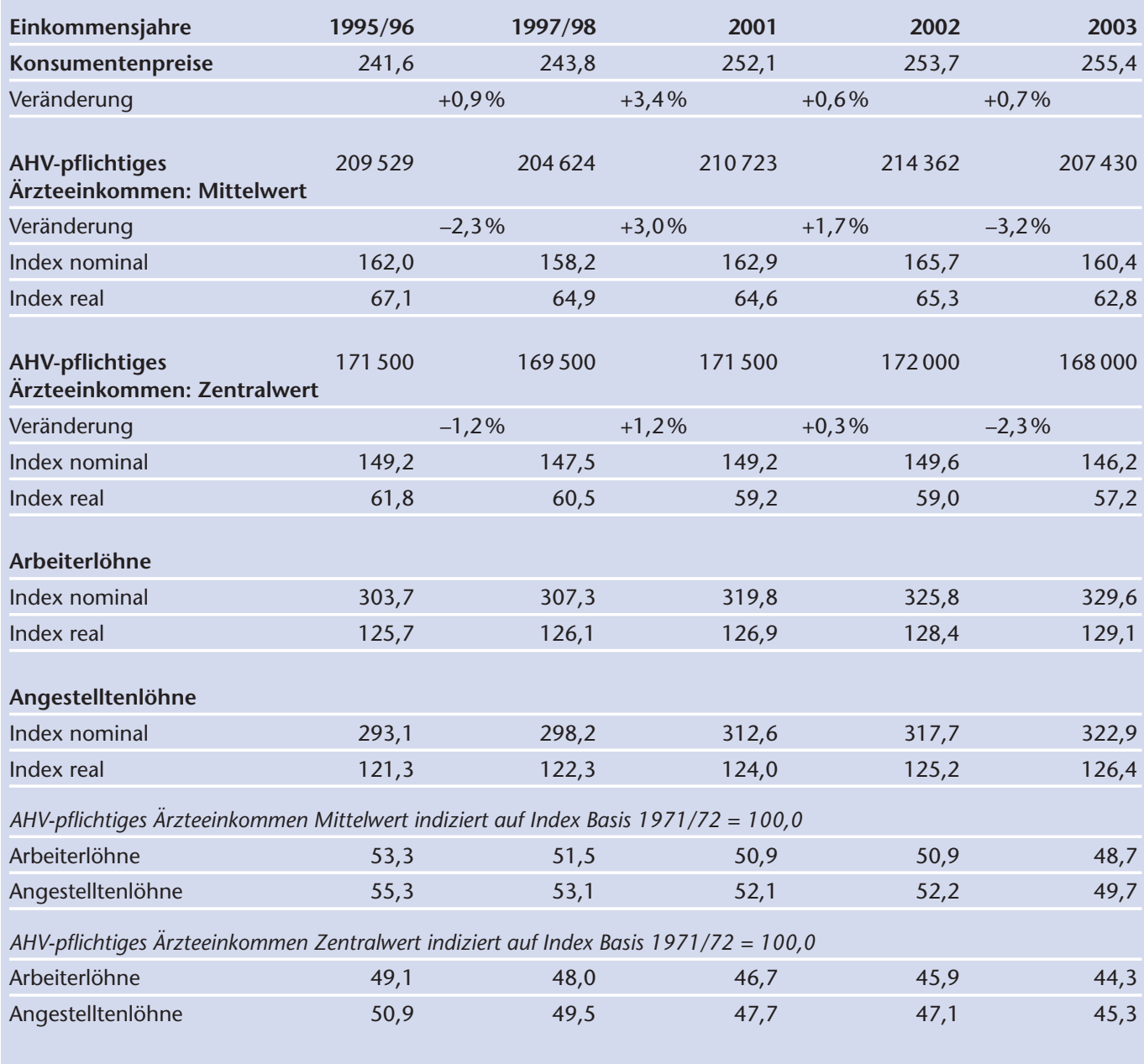


Abbildung 13

Entwicklung des Mittelwertes des AHV-pflichtigen Einkommens der freien Ärzteschaft der Schweiz unter 66 Jahren (nominal) $1971 / 72$ bis 2003 .

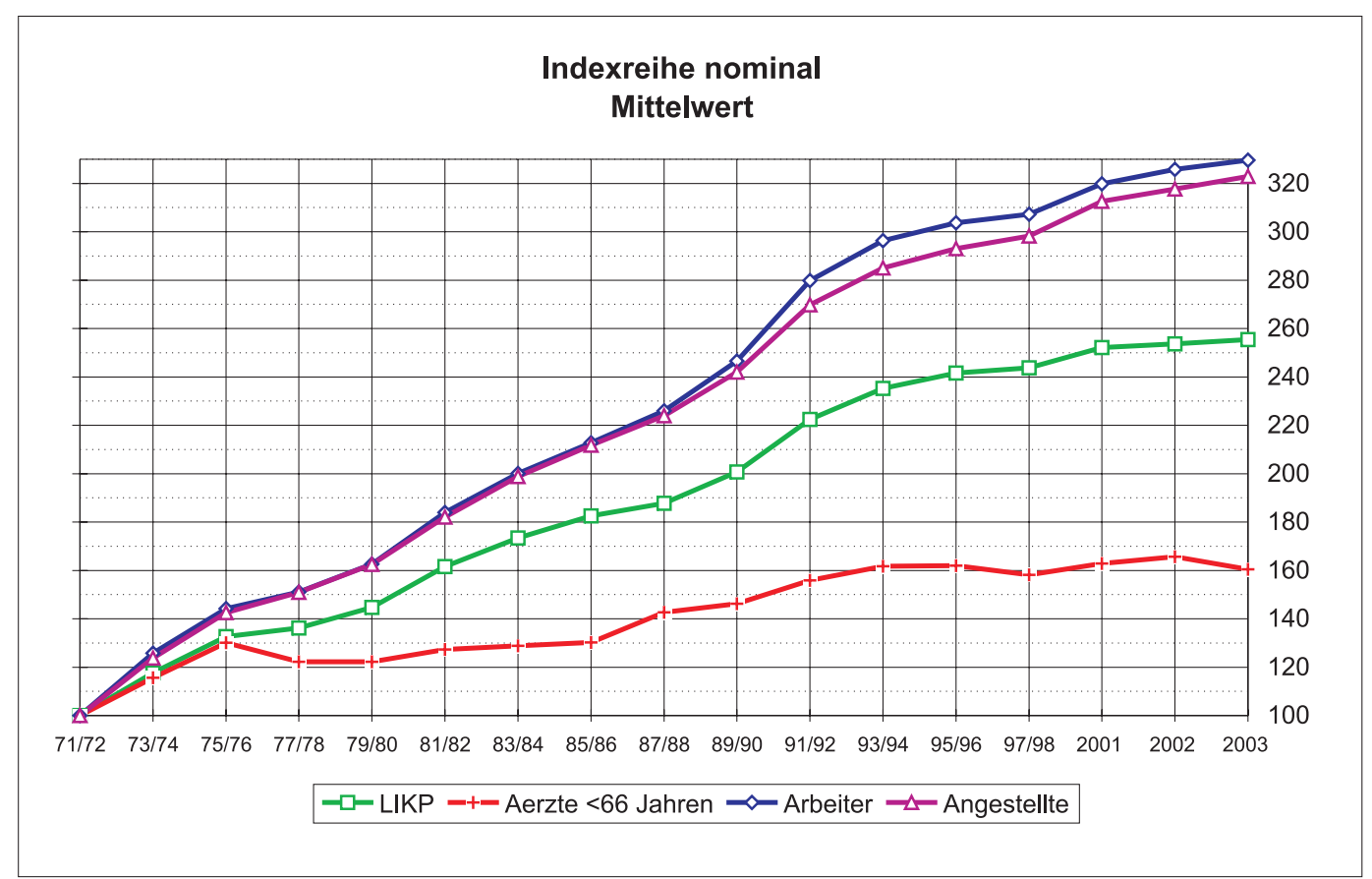

\section{Abbildung 14}

Entwicklung des Mittelwertes des AHV-pflichtigen Einkommens der freien Ärzteschaft der Schweiz unter 66 Jahren (real) $1971 / 72$ bis 2003.

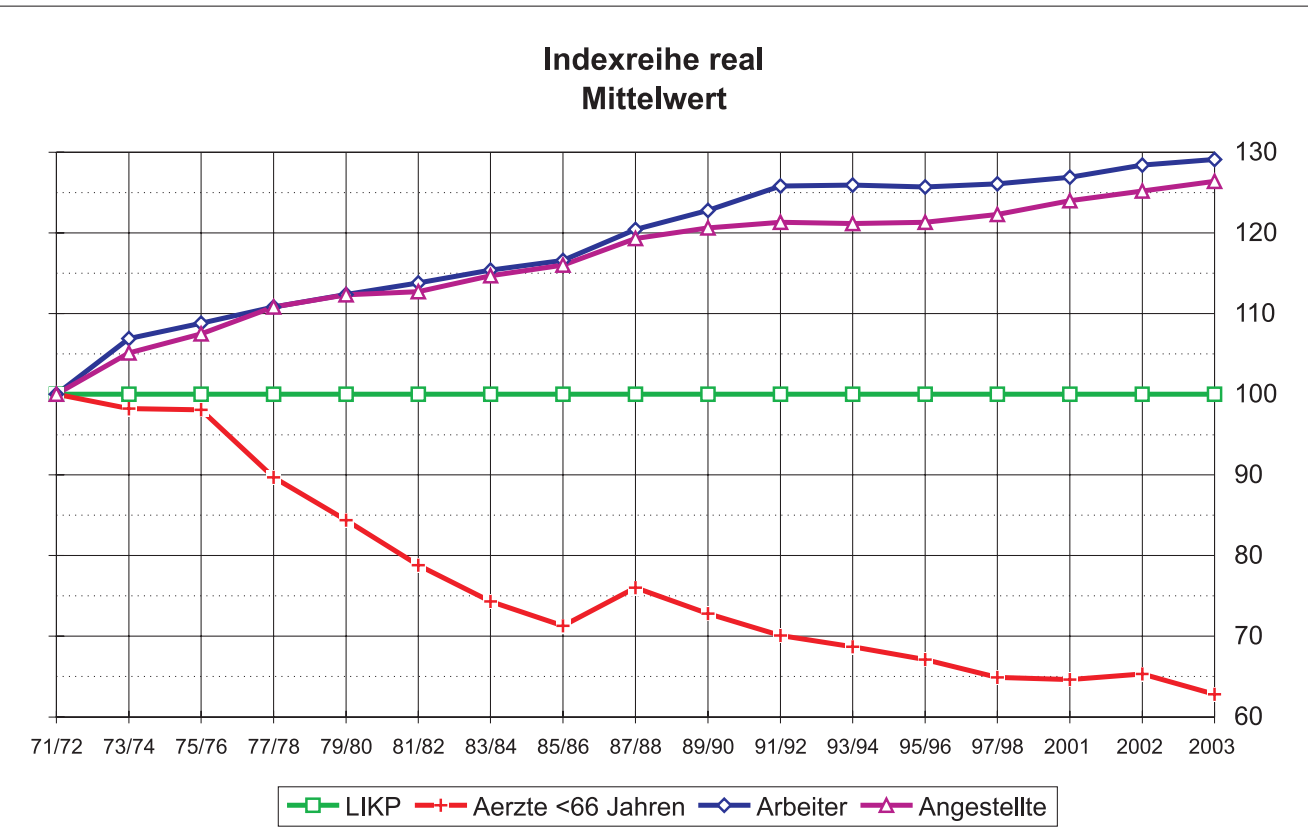


Abbildung 15

Entwicklung des Zentralwertes des AHV-pflichtigen Einkommens der freien Ärzteschaft der Schweiz unter 66 Jahren (nominal) 1971/72 bis 2003.

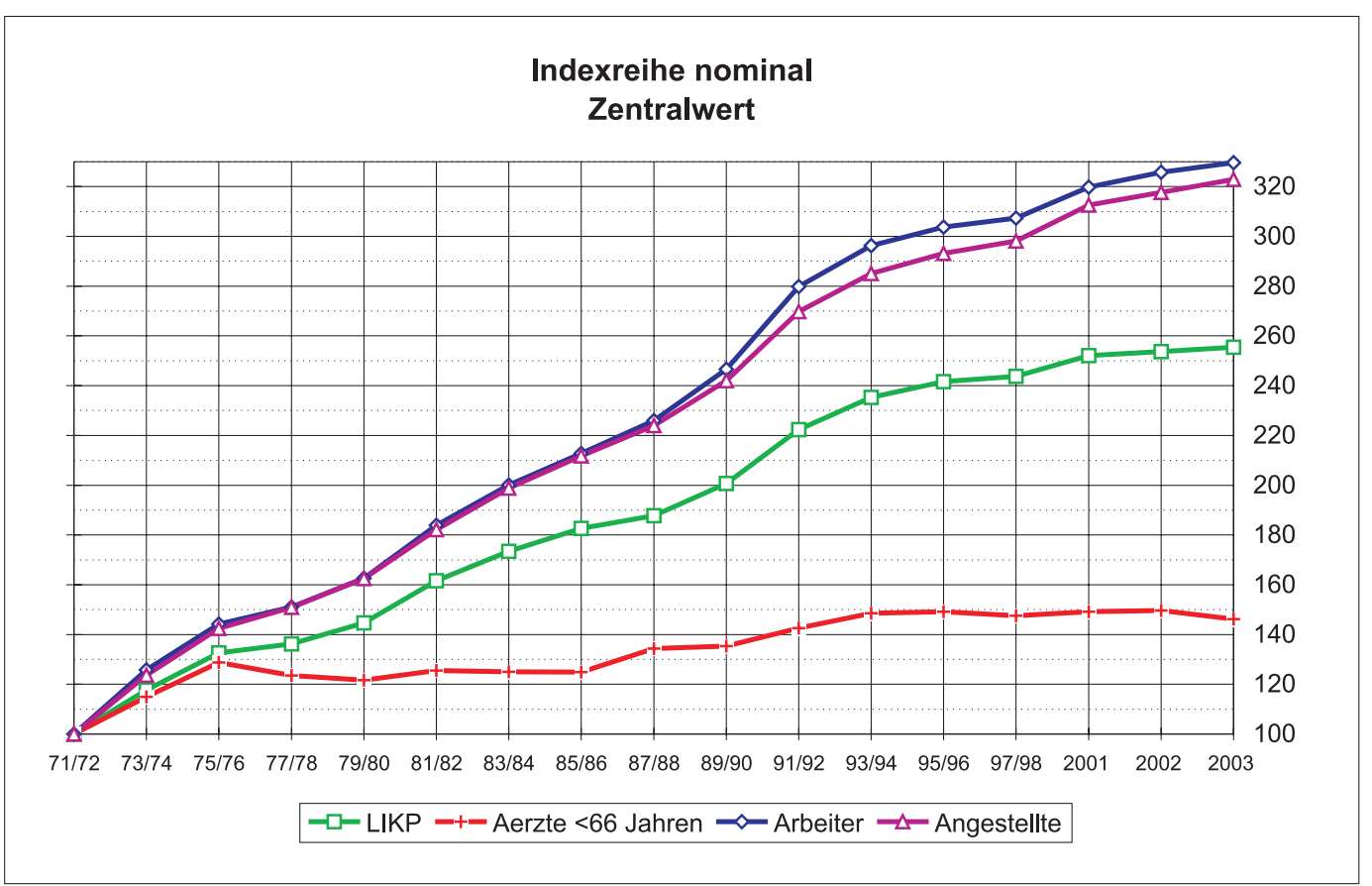

\section{Abbildung 16}

Entwicklung des Zentralwertes des AHV-pflichtigen Einkommens der freien Ärzteschaft der Schweiz unter 66 Jahren (real) $1971 / 72$ bis 2003 .

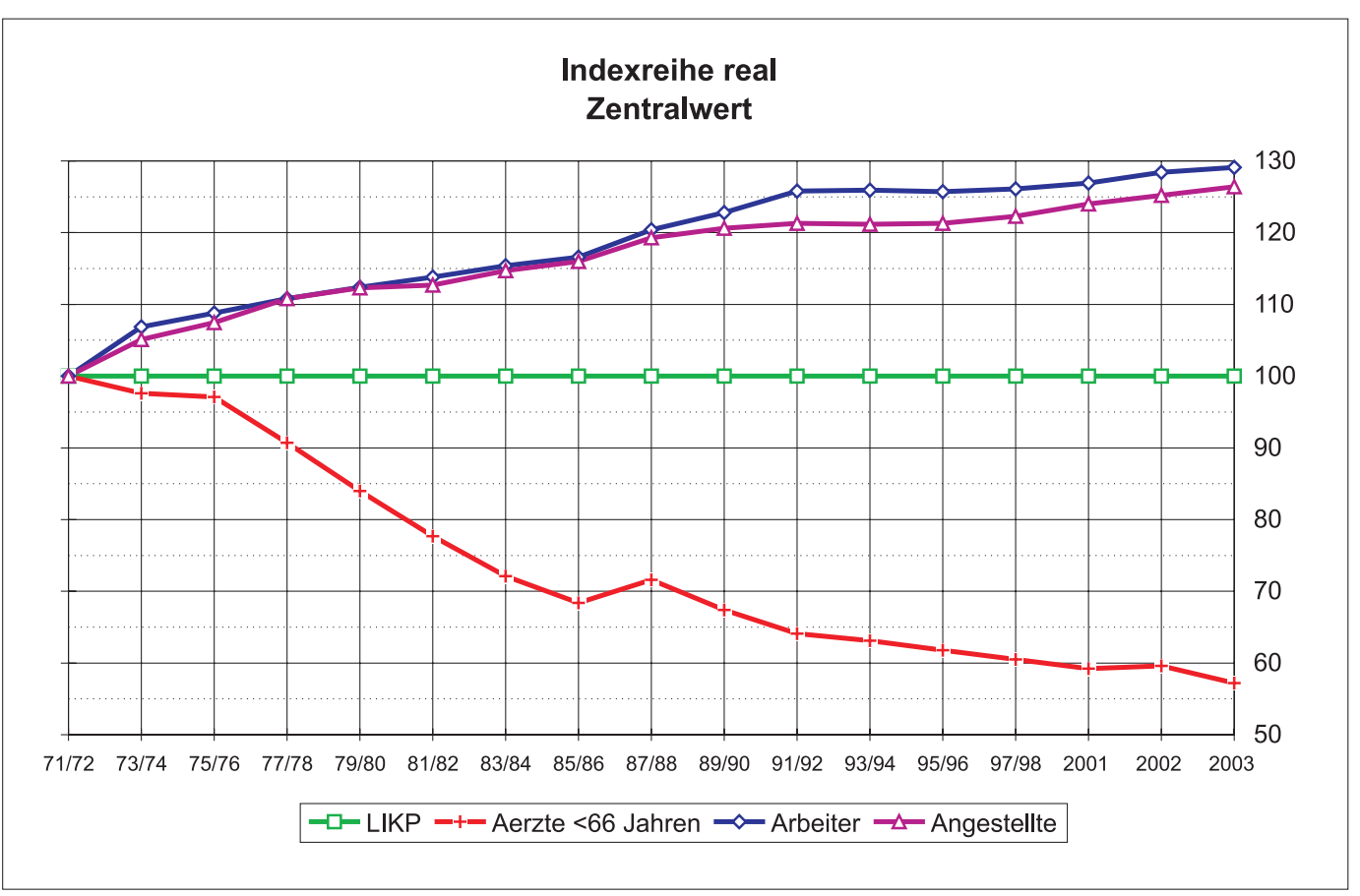


Tabelle 5

AHV-pflichtiges Einkommen aller in freier Praxis tätigen Ärzte. Mittelwerte 2002 und 2003 nach Kantonen,

Ärzte unter 66 Jahren.

\begin{tabular}{|c|c|c|c|c|c|c|c|}
\hline Kanton & Anzahl & 2002 & Index & $+/-$ & Anzahl & 2003 & Index \\
\hline $\mathrm{ZH}$ & 2480 & 217255 & $101,3 \%$ & $-4,6 \%$ & 1791 & 207200 & $99,9 \%$ \\
\hline $\mathrm{BE}$ & 1793 & 209427 & $97,7 \%$ & $-2,6 \%$ & 1639 & 203934 & $98,3 \%$ \\
\hline LU & 417 & 237024 & $110,6 \%$ & $-1,5 \%$ & 378 & 233512 & $112,6 \%$ \\
\hline UR & 32 & 185094 & $86,3 \%$ & $+10,1 \%$ & 29 & 203859 & $98,3 \%$ \\
\hline SZ & 131 & 257400 & $120,1 \%$ & $-19,4 \%$ & 46 & 207448 & $100,0 \%$ \\
\hline UW & 74 & 213469 & $99,6 \%$ & $+3,6 \%$ & 69 & 221191 & $106,6 \%$ \\
\hline GL & 40 & 276108 & $128,8 \%$ & $-8,2 \%$ & 42 & 253405 & $122,2 \%$ \\
\hline ZG & 154 & 227966 & $106,3 \%$ & $-4,8 \%$ & 157 & 217044 & $104,6 \%$ \\
\hline FR & 338 & 224107 & $104,5 \%$ & $-1,9 \%$ & 347 & 219748 & $105,9 \%$ \\
\hline SO & 378 & 215867 & $100,7 \%$ & $-2,7 \%$ & 358 & 210104 & $101,3 \%$ \\
\hline BS & 469 & 176275 & $82,2 \%$ & $-2,0 \%$ & 439 & 172759 & $83,3 \%$ \\
\hline$B L$ & 482 & 240840 & $112,4 \%$ & $-5,3 \%$ & 473 & 228159 & $110,0 \%$ \\
\hline SH & 128 & 205339 & $95,8 \%$ & $-4,4 \%$ & 131 & 196252 & $94,6 \%$ \\
\hline AP & 100 & 205600 & $95,9 \%$ & $+9,6 \%$ & 87 & 225329 & $108,6 \%$ \\
\hline SG & 523 & 218168 & $101,8 \%$ & $+1,3 \%$ & 514 & 220936 & $106,5 \%$ \\
\hline GR & 284 & 194221 & $90,6 \%$ & $-2,0 \%$ & 247 & 190340 & $91,8 \%$ \\
\hline AG & 650 & 216010 & $100,8 \%$ & $+4,0 \%$ & 504 & 224731 & $108,3 \%$ \\
\hline TG & 295 & 232669 & $108,5 \%$ & $-4,7 \%$ & 295 & 221737 & $106,9 \%$ \\
\hline TI & 463 & 252480 & $117,8 \%$ & $-6,9 \%$ & 63 & 235117 & $113,3 \%$ \\
\hline VD & 884 & 219044 & $102,2 \%$ & $-1,5 \%$ & 745 & 215663 & $104,0 \%$ \\
\hline VS & 339 & 175142 & $81,7 \%$ & $-5,9 \%$ & 325 & 164833 & $79,5 \%$ \\
\hline NE & 171 & 148171 & $69,1 \%$ & $+3,0 \%$ & 160 & 152554 & $73,5 \%$ \\
\hline GE & 199 & 174857 & $81,6 \%$ & $+4,0 \%$ & 178 & 181836 & $87,7 \%$ \\
\hline JU & 80 & 142293 & $66,4 \%$ & $+2,2 \%$ & 76 & 145479 & $70,1 \%$ \\
\hline Schweiz & 10904 & 214362 & $100,0 \%$ & $-3,2 \%$ & 9093 & 207430 & $100,0 \%$ \\
\hline
\end{tabular}

Tabelle 9

AHV-pflichtiges Einkommen aller in freier Praxis tätigen Ärzte, Mittelwerte 2002 und 2003 nach Spezialitäten,

Ärzte unter 66 Jahren.

\begin{tabular}{|c|c|c|c|c|c|c|c|}
\hline Spezialität & Anzahl & 2002 & Index & $+/-$ & Anzahl & 2003 & Index \\
\hline Anästhesie & 297 & 276525 & $129,0 \%$ & $-6,2 \%$ & 241 & 259398 & $125,1 \%$ \\
\hline Chirurgie & 537 & 273131 & $127,4 \%$ & $-8,3 \%$ & 411 & 250431 & $120,7 \%$ \\
\hline Dermatologie & 218 & 252373 & $117,7 \%$ & $-2,9 \%$ & 173 & 245171 & $118,2 \%$ \\
\hline Gynäkologie & 682 & 253028 & $118,0 \%$ & $+0,5 \%$ & 543 & 254413 & $122,7 \%$ \\
\hline Innere Medizin & 2118 & 218696 & $102,0 \%$ & $-3,8 \%$ & 1729 & 210303 & $101,4 \%$ \\
\hline Neurochirurgie & 29 & 346383 & $161,6 \%$ & $-10,8 \%$ & 22 & 309145 & $149,0 \%$ \\
\hline Neurologie & 137 & 188215 & $87,8 \%$ & $-9,1 \%$ & 108 & 171030 & $82,5 \%$ \\
\hline Ophthalmologie & 386 & 378000 & $176,3 \%$ & $-1,8 \%$ & 325 & 371361 & $179,0 \%$ \\
\hline Orthopädie & 267 & 298640 & $139,3 \%$ & $+0,1 \%$ & 220 & 298930 & $144,1 \%$ \\
\hline ORL & 188 & 297732 & $138,9 \%$ & $-4,2 \%$ & 164 & 285249 & $137,5 \%$ \\
\hline Pädiatrie & 526 & 169334 & $79,0 \%$ & $+0,5 \%$ & 430 & 170213 & $82,1 \%$ \\
\hline $\begin{array}{l}\text { Physikalische } \\
\text { Medizin }\end{array}$ & 161 & 218065 & $101,7 \%$ & $-11,1 \%$ & 135 & 193921 & $93,5 \%$ \\
\hline Psychiatrie & 1317 & 118940 & $55,5 \%$ & $-0,8 \%$ & 1154 & 117943 & $56,9 \%$ \\
\hline Kinderpsychiatrie & 194 & 106443 & $49,7 \%$ & $+2,3 \%$ & 177 & 108852 & $52,5 \%$ \\
\hline
\end{tabular}




\begin{tabular}{|c|c|c|c|c|c|c|c|}
\hline Radiologie & 141 & 398102 & $185,7 \%$ & $-0,9 \%$ & 118 & 394586 & $190,2 \%$ \\
\hline Urologie & 97 & 339214 & $158,2 \%$ & $-3,4 \%$ & 82 & 327529 & $157,9 \%$ \\
\hline Allgemeinmedizin & 3596 & 193844 & $90,4 \%$ & $-2,3 \%$ & 3046 & 189424 & $91,3 \%$ \\
\hline Spezialisten & 7308 & 224457 & $104,7 \%$ & $-3,5 \%$ & 6047 & 216501 & $104,4 \%$ \\
\hline Allgemeinmedizin & 3596 & 193844 & $90,4 \%$ & $-2,3 \%$ & 3046 & 189424 & $91,3 \%$ \\
\hline Alle & 10904 & 214362 & $100,0 \%$ & $-3,2 \%$ & 9093 & 207430 & $100,0 \%$ \\
\hline
\end{tabular}

Tabelle 7

AHV-pflichtiges Einkommen aller in freier Praxis tätigen Ärzte, Zentralwerte 2002 und 2003 nach Kantonen, Ärzte unter 66 Jahren.

\begin{tabular}{|c|c|c|c|c|c|c|c|}
\hline Kanton & Anzahl & 2002 & Index & $+/-$ & Anzahl & 2003 & Index \\
\hline $\mathrm{ZH}$ & 2480 & 162700 & $94,6 \%$ & $-3,1 \%$ & 1791 & 157700 & $93,9 \%$ \\
\hline $\mathrm{BE}$ & 1793 & 165900 & $96,5 \%$ & $-1,3 \%$ & 1639 & 163800 & $97,5 \%$ \\
\hline LU & 417 & 203400 & $118,3 \%$ & $+1,0 \%$ & 378 & 205500 & $122,3 \%$ \\
\hline UR & 32 & 190800 & $110,9 \%$ & $-6,3 \%$ & 29 & 178700 & $106,4 \%$ \\
\hline SZ & 131 & 236000 & $137,2 \%$ & $-20,8 \%$ & 46 & 186800 & $111,2 \%$ \\
\hline UW & 74 & 193800 & $112,7 \%$ & $-8,3 \%$ & 69 & 177800 & $105,8 \%$ \\
\hline $\mathrm{GL}$ & 40 & 260700 & $151,6 \%$ & $-3,5 \%$ & 42 & 251500 & $149,7 \%$ \\
\hline ZG & 154 & 224800 & $130,7 \%$ & $-4,2 \%$ & 157 & 215300 & $128,2 \%$ \\
\hline FR & 338 & 192100 & $111,7 \%$ & $-0,8 \%$ & 347 & 190600 & $113,5 \%$ \\
\hline SO & 378 & 182900 & $106,3 \%$ & $-6,0 \%$ & 358 & 172000 & $102,4 \%$ \\
\hline BS & 469 & 133800 & $77,8 \%$ & $-1,7 \%$ & 439 & 131500 & $78,3 \%$ \\
\hline$B L$ & 482 & 190900 & $111,0 \%$ & $-5,7 \%$ & 473 & 180000 & $107,1 \%$ \\
\hline $\mathrm{SH}$ & 128 & 155400 & $90,3 \%$ & $-2,5 \%$ & 131 & 151500 & $90,2 \%$ \\
\hline AP & 100 & 167200 & $97,2 \%$ & $+2,0 \%$ & 87 & 170600 & $101,5 \%$ \\
\hline SG & 523 & 188600 & $109,7 \%$ & $+2,7 \%$ & 514 & 193600 & $115,2 \%$ \\
\hline GR & 284 & 162400 & $94,4 \%$ & $+0,1 \%$ & 247 & 162600 & $96,8 \%$ \\
\hline$A G$ & 650 & 177300 & $103,1 \%$ & $+0,5 \%$ & 504 & 178100 & $106,0 \%$ \\
\hline TG & 295 & 197700 & $114,9 \%$ & $-4,0 \%$ & 295 & 189700 & $112,9 \%$ \\
\hline TI & 463 & 208700 & $121,3 \%$ & $-5,7 \%$ & 63 & 196900 & $117,2 \%$ \\
\hline VD & 884 & 182700 & $106,2 \%$ & $-3,1 \%$ & 745 & 177000 & $105,4 \%$ \\
\hline VS & 339 & 153600 & $89,3 \%$ & $-7,2 \%$ & 325 & 142500 & $84,8 \%$ \\
\hline $\mathrm{NE}$ & 171 & 126600 & $73,6 \%$ & $+2,0 \%$ & 160 & 129100 & $76,8 \%$ \\
\hline GE & 199 & 135500 & $78,8 \%$ & $+0,8 \%$ & 178 & 136600 & $81,3 \%$ \\
\hline JU & 80 & 137000 & $79,7 \%$ & $+10,7 \%$ & 76 & 151600 & $90,2 \%$ \\
\hline Schweiz & 10904 & 172000 & $100,0 \%$ & $-2,3 \%$ & 9093 & 168000 & $100,0 \%$ \\
\hline
\end{tabular}


Tabelle 13

AHV-pflichtiges Einkommen aller in freier Praxis tätigen Ärzte, Zentralwerte 2002 und 2003 nach Spezialitäten, Ärzte unter 66 Jahren.

\begin{tabular}{|c|c|c|c|c|c|c|c|}
\hline Spezialität & Anzahl & 2002 & Index & $+/-$ & Anzahl & 2003 & Index \\
\hline Anästhesie & 297 & 227500 & $132,3 \%$ & $+8,4 \%$ & 241 & 246600 & $146,8 \%$ \\
\hline Chirurgie & 537 & 206900 & $120,3 \%$ & $-6,7 \%$ & 411 & 193000 & $114,9 \%$ \\
\hline Dermatologie & 218 & 193300 & $112,4 \%$ & $+7,6 \%$ & 173 & 207900 & $123,8 \%$ \\
\hline Gynäkologie & 682 & 220400 & $128,1 \%$ & $-4,4 \%$ & 543 & 210700 & $125,4 \%$ \\
\hline Innere Medizin & 2118 & 184000 & $107,0 \%$ & $-1,9 \%$ & 1729 & 180500 & $107,4 \%$ \\
\hline Neurochirurgie & 29 & 250600 & $145,7 \%$ & $-41,4 \%$ & 22 & 146900 & $87,4 \%$ \\
\hline Neurologie & 137 & 148000 & $86,0 \%$ & $-1,4 \%$ & 108 & 146000 & $86,9 \%$ \\
\hline Ophthalmologie & 386 & 271200 & $157,7 \%$ & $-16,4 \%$ & 325 & 226600 & $134,9 \%$ \\
\hline Orthopädie & 267 & 239900 & $139,5 \%$ & $+5,7 \%$ & 220 & 253500 & $150,9 \%$ \\
\hline ORL & 188 & 249600 & $145,1 \%$ & $+5,6 \%$ & 164 & 263600 & $156,9 \%$ \\
\hline Pädiatrie & 526 & 146500 & $85,2 \%$ & $+7,2 \%$ & 430 & 157100 & $93,5 \%$ \\
\hline $\begin{array}{l}\text { Physikalische } \\
\text { Medizin }\end{array}$ & 161 & 197300 & $114,7 \%$ & $-11,4 \%$ & 135 & 174900 & $104,1 \%$ \\
\hline Psychiatrie & 1317 & 112000 & $65,1 \%$ & $+0,4 \%$ & 1154 & 112500 & $67,0 \%$ \\
\hline Kinderpsychiatrie & 194 & 107000 & $62,2 \%$ & $+0,7 \%$ & 177 & 107700 & $64,1 \%$ \\
\hline Radiologie & 141 & 294700 & $171,3 \%$ & $+2,3 \%$ & 118 & 301500 & $179,5 \%$ \\
\hline Urologie & 97 & 264000 & $153,5 \%$ & $+17,8 \%$ & 82 & 311100 & $185,2 \%$ \\
\hline Allgemeinmedizin & 3596 & 175400 & $102,0 \%$ & $-1,4 \%$ & 3046 & 172900 & $102,9 \%$ \\
\hline Spezialisten & 7308 & 170300 & $99,0 \%$ & $-3,1 \%$ & 6047 & 165100 & $98,3 \%$ \\
\hline Allgemeinmedizin & 3596 & 175400 & $102,0 \%$ & $-1,4 \%$ & 3046 & 172900 & $102,9 \%$ \\
\hline Alle & 10904 & 172000 & $100,0 \%$ & $-2,3 \%$ & 9093 & 168000 & $100,0 \%$ \\
\hline
\end{tabular}

